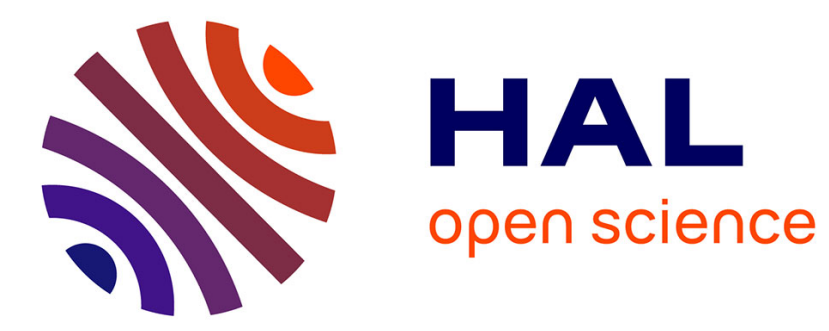

\title{
Intensities and spatiotemporal variability of equatorial noise emissions observed by the Cluster spacecraft
}

\author{
F. Nemec, O. Santolík, Z. Hrbackova, Nicole Cornilleau-Wehrlin
}

\section{To cite this version:}

F. Nemec, O. Santolík, Z. Hrbackova, Nicole Cornilleau-Wehrlin. Intensities and spatiotemporal variability of equatorial noise emissions observed by the Cluster spacecraft. Journal of Geophysical Research Space Physics, 2015, 120, pp.1620-1632. 10.1002/2014JA020814 . hal-01551998

\author{
HAL Id: hal-01551998 \\ https://hal.science/hal-01551998
}

Submitted on 29 Nov 2021

HAL is a multi-disciplinary open access archive for the deposit and dissemination of scientific research documents, whether they are published or not. The documents may come from teaching and research institutions in France or abroad, or from public or private research centers.
L'archive ouverte pluridisciplinaire HAL, est destinée au dépôt et à la diffusion de documents scientifiques de niveau recherche, publiés ou non, émanant des établissements d'enseignement et de recherche français ou étrangers, des laboratoires publics ou privés.

$$
\text { Copyright }
$$




\section{Journal of Geophysical Research: Space Physics}

\section{RESEARCH ARTICLE \\ 10.1002/2014JA020814 \\ Key Points: \\ Intensities and spatiotemporal variability of equatorial noise emissions observed by the Cluster spacecraft}

- Survey of more than 2000

equatorial noise events observed

by Cluster spacecraft

- Intensity versus frequency,

plasmasphere/plasma trough, MLT,

and geomagnetic activity

- Spatiotemporal variability

investigated using

multipoint measurements

\section{Correspondence to:}

F. Němec,

frantisek.nemec@gmail.com

\section{Citation:}

Němec, F., O. Santolík, Z. Hrbáčková, and N. Cornilleau-Wehrlin (2015), Intensities and spatiotemporal variability of equatorial noise emissions observed by the Cluster spacecraft, J. Geophys. Res. Space Physics, 120, 1620-1632, doi:10.1002/2014JA020814.

Received 10 NOV 2014 Accepted 30 JAN 2015

Accepted article online 4 FEB 2015 Published online 5 MAR 2015

\author{
F. Němec ${ }^{1}$, O. Santolík ${ }^{1,2}$, Z. Hrbáčková ${ }^{1,2}$, and N. Cornilleau-Wehrlin ${ }^{3,4}$ \\ ${ }^{1}$ Faculty of Mathematics and Physics, Charles University in Prague, Prague, Czech Republic, ${ }^{2}$ Institute of Atmospheric \\ Physics, Academy of Sciences of the Czech Republic, Prague, Czech Republic, ${ }^{3}$ Laboratoire de Physique des Plasmas, Ecole \\ Polytechnique, CNRS, Palaiseau, France, ${ }^{4}$ LESIA, Observatoire de Meudon, Meudon, France
}

\section{Introduction}

Equatorial noise (EN) emissions are electromagnetic waves at frequencies between the proton cyclotron frequency and the lower hybrid frequency observed in the inner magnetosphere at radial distances between about 2 and $7 R_{E}$ and latitudes within about $10^{\circ}$ from the geomagnetic equator [Laakso et al., 1990; Kasahara et al., 1994]. They were reported for the first time by Russell et al. [1970], who called them "noise" because of their apparent lack of inner structure in the low-resolution OGO 3 data. They were shown to have the magnetic field fluctuations nearly linearly polarized along the ambient magnetic field, corresponding to the propagation in the extraordinary mode nearly perpendicular to the ambient magnetic field [Stix, 1992]. In the low-frequency approximation this corresponds to the fast magnetosonic mode, and EN emissions are thus sometimes called "magnetosonic waves."

Higher-resolution measurements of EN by the IMP 6 and the Hawkeye 1 satellites revealed that EN consists of a complex superposition of many harmonically spaced lines [Gurnett, 1976]. The frequency structure is assumed to be linked to the proton cyclotron frequency in the source region. EN emissions are believed to be generated by energetic protons with ring-like distribution functions [Curtis and Wu, 1979; Perraut et al., 1982; McClements and Dendy, 1993; McClements et al., 1994; Horne et al., 2000; Liu et al., 2011; Chen et al., 2011] observed in association with the waves [Perraut et al., 1982; Boardsen et al., 1992; Xiao et al., 2013; Ma et al., 2014]. Meredith et al. [2008] statistically analyzed the relationship between the distribution of the wave electric field intensity and the ion ring distributions using the data obtained by the Combined Release and Radiation Effects Satellite (CRRES). They found a close match between the magnetic local time (MLT) distribution of low-energy proton rings $(<30 \mathrm{keV}$ ) exceeding the instability threshold and the distribution of the wave intensity on the dusk side, while the situation on the dawn side was far less clear. Chen et al. [2010] simulated the global distribution of EN using modeled proton ring distributions. They showed that low harmonic waves tend to be excited in the high-density nightside plasmasphere, whereas higher-frequency 
waves are excited over a broad spatial region of low density outside the morningside plasmasphere. The relationship between the plasma number density in the source region and the generated harmonics was further confirmed by Ma et al. [2014].

High-resolution measurements of EN emissions by the Wide-Band Data (WBD) instruments on board the Cluster spacecraft along with the spacecraft rotation and theoretical polarization properties of the emissions were used by Santolik et al. [2002] to determine the azimuthal direction of propagation of the observed emissions. The same approach was used by Němec et al. [2013] to determine the directions of propagation of all EN events observed by the Cluster spacecraft during the first 10 years of operation for which the WBD data were available. They found that while principally isotropic distribution of wave vector directions in the equatorial plane is detected inside the plasmasphere, wave propagation in the plasma trough is predominantly directed to the west or to the east. They concluded that the observed propagation pattern is in agreement with the propagation analysis of Chen and Thorne [2012], assuming that the emissions are generated close to the plasmapause. Raytracing studies of EN emissions further show that EN emissions can propagate over a broad region of MLTs, particularly inside the plasmasphere, and cross the plasmapause boundary [Kasahara et al., 1994; Xiao et al., 2012].

EN is the most intense natural electromagnetic emission at latitudes within about $10^{\circ}$ from the geomagnetic equator and frequencies between the proton cyclotron frequency and the lower hybrid frequency. It could thus play a nonnegligible role in the dynamics of the inner magnetosphere [Santolík et al., 2004]. EN interaction with radiation belt electrons has been recently discussed by several authors [Horne et al., 2007; Bortnik and Thorne, 2010; Shprits et al., 2013; Mourenas et al., 2013; Li et al., 2014], which documents their potential significance for electron acceleration and loss. Additionally, systematic surveys of EN occurrence and intensities have been presented [Ma et al., 2013; Tsurutani et al., 2014; Hrbáčková et al., 2015], indicating an increased interest in this electromagnetic emission.

The present study uses a set of 2229 EN events identified in the first 10 years of the Cluster Spatio-Temporal Analysis of Field Fluctuations spectrum analyzer (STAFF-SA) data [Hrbáčková et al., 2015] to analyze their intensity as a function of relevant parameters. Exact frequency-time intervals of EN emissions are identified using fuzzy logic based on wave propagation parameters, rather than only on the electric/magnetic field intensity and the frequency range. Moreover, the plasmasphere and the plasma trough regions are classified using the estimated plasma number densities at the observation points. We also analyze the spatiotemporal variability of the emissions using multipoint observations. Section 2 describes the data set used in the study. The obtained results are described in section 3 and discussed in section 4 . Finally, section 5 contains a brief summary.

\section{Data Set}

Multicomponent electromagnetic wave data measured by the four Cluster spacecraft have been used. The Cluster spacecraft have nearly identical elliptical orbits. The orbital parameters have changed over the duration of the mission, but during the first years of operation the apogee was at an altitude of about $119,000 \mathrm{~km}$ and the perigee was about $24,000 \mathrm{~km}$. Multicomponent measurements of electromagnetic waves are performed by the STAFF-SA instrument. Three orthogonal magnetic field components and two electric field components in the spin plane of the spacecraft are used for onboard calculation of $5 \times 5$ complex spectral matrices. The analysis is limited to 27 logarithmically spaced frequency channels between $8 \mathrm{~Hz}$ and $4 \mathrm{kHz}$. One spectral matrix per frequency channel and time interval is obtained, which can be used to determine not only the intensity of observed electromagnetic waves but also their polarization and propagation properties. A detailed description of the instrumentation can be found in Cornilleau-Wehrlin et al. [1997, 2003].

We have used the list of 2229 EN events compiled by Hrbáčková et al. [2015] based on the data measured by the Cluster spacecraft during the first 10 years of the operation (2001-2010). The list provides us with the beginning and ending times of EN events that are used for further analysis.

Examples of EN emissions observed by the Cluster spacecraft are shown in Figures 1 and 2. They correspond to the same EN event, but they were observed by two different Cluster spacecraft with a small time and spatial separation. The data plotted in Figure 1 were measured by Cluster 1 on 24 November 2004 between 0900:00 and 0948:00 UT. The data plotted in Figure 2 were measured by Cluster 2 on the same day between 0904:00 and 0945:00 UT. Both figures have the same format. The first and second panels show 


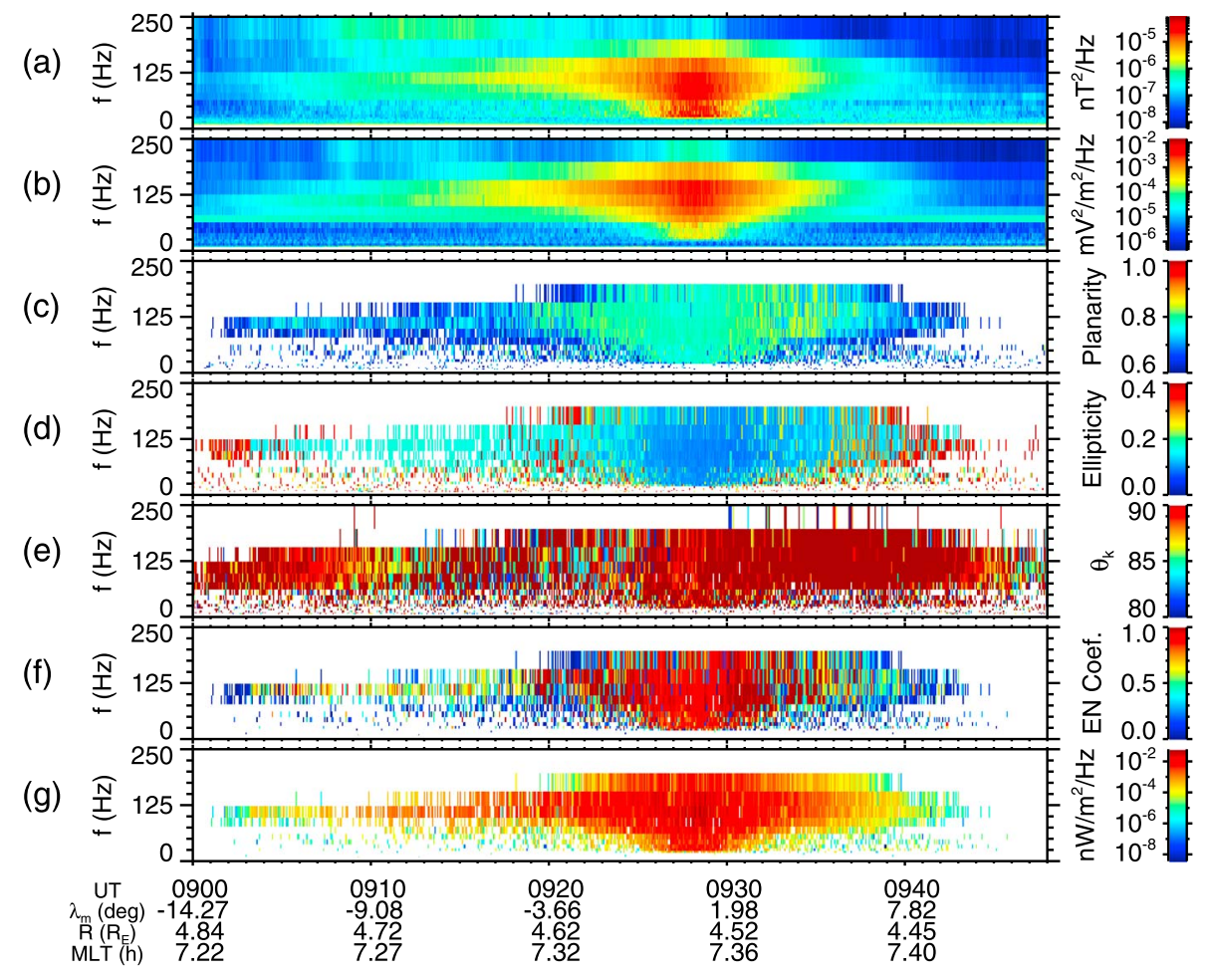

Figure 1. Example of an equatorial noise event observed by Cluster 1, along with the related wave analysis. The data were measured on 24 November 2004 between 0900:00 and 0948:00 UT: (a) frequency-time spectrogram of power spectral density of magnetic field fluctuations, (b) frequency-time spectrogram of power spectral density of electric field fluctuations, (c) frequency-time plot of planarity of magnetic field fluctuations, (d) frequency-time plot of ellipticity of magnetic field fluctuations, (e) frequency-time plot of wave normal angle, (f) frequency-time plot of EN coefficient (see text), and (g) frequency-time plot of estimated EN Poynting flux spectral density (see text).

the frequency-time spectrograms of power spectral density of magnetic and electric field fluctuations, respectively. EN are the intense emissions with the maximum intensity in the middle of the plotted time interval in Figure 1 and toward the end of the plotted time interval in Figure 2.

Figures $1 \mathrm{c}$ and $2 \mathrm{c}$ show the frequency-time plots of the planarity of magnetic field fluctuations calculated using the singular value decomposition (SVD) method [Santolik et al., 2003]. Only frequency-time intervals with the values of planarity larger than 0.6 are plotted. The planarity of EN emissions is typically above 0.8 [Santolik et al., 2004], and following Němec et al. [2013], we will use this planarity threshold for identification of EN frequency-time intervals. Figures $1 d$ and $2 d$ show the frequency-time plots of the ellipticity of magnetic field fluctuations calculated using the SVD method of Santolik et al. [2003]. Only frequency-time intervals with the values of ellipticity lower than 0.4 are plotted. EN emissions are nearly linearly polarized, and following Santolík et al. [2004], Němec et al. [2013], and Hrbáčková et al. [2015], we will use an ellipticity threshold of 0.2 for identification of EN frequency-time intervals. Figures 1e and 2e show the frequency-time plots of the wave normal angles determined using the SVD method of Santolik et al. [2003] from the magnetic parts of the spectral matrices. Only frequency-time intervals with the values of wave normal angles larger than $80^{\circ}$ are plotted. EN emissions have wave normal angles close to $90^{\circ}$; i.e., the wave vectors are oriented nearly perpendicular to the ambient magnetic field. Following Němec et al. [2013], we will use a threshold of $85^{\circ}$ for wave normal angle.

It should be noted that these thresholds are not independent, but in principle they are strictly related by the polarization and propagation properties of electromagnetic waves in the plasma environment [Stix, 1992]. However, as the real data always contain some amount of noise, which enters differently in the calculation of various parameters, it proves to be efficient to use the three thresholds simultaneously [Němec et al., 2013]. Moreover, as the point of the present analysis is to obtain the intensity of EN emissions in a given frequency-time interval, a binary logic ("EN is observed or EN is not observed in a given frequency-time interval") turns out to be far from optimal. A better approach might be to use a simple fuzzy 


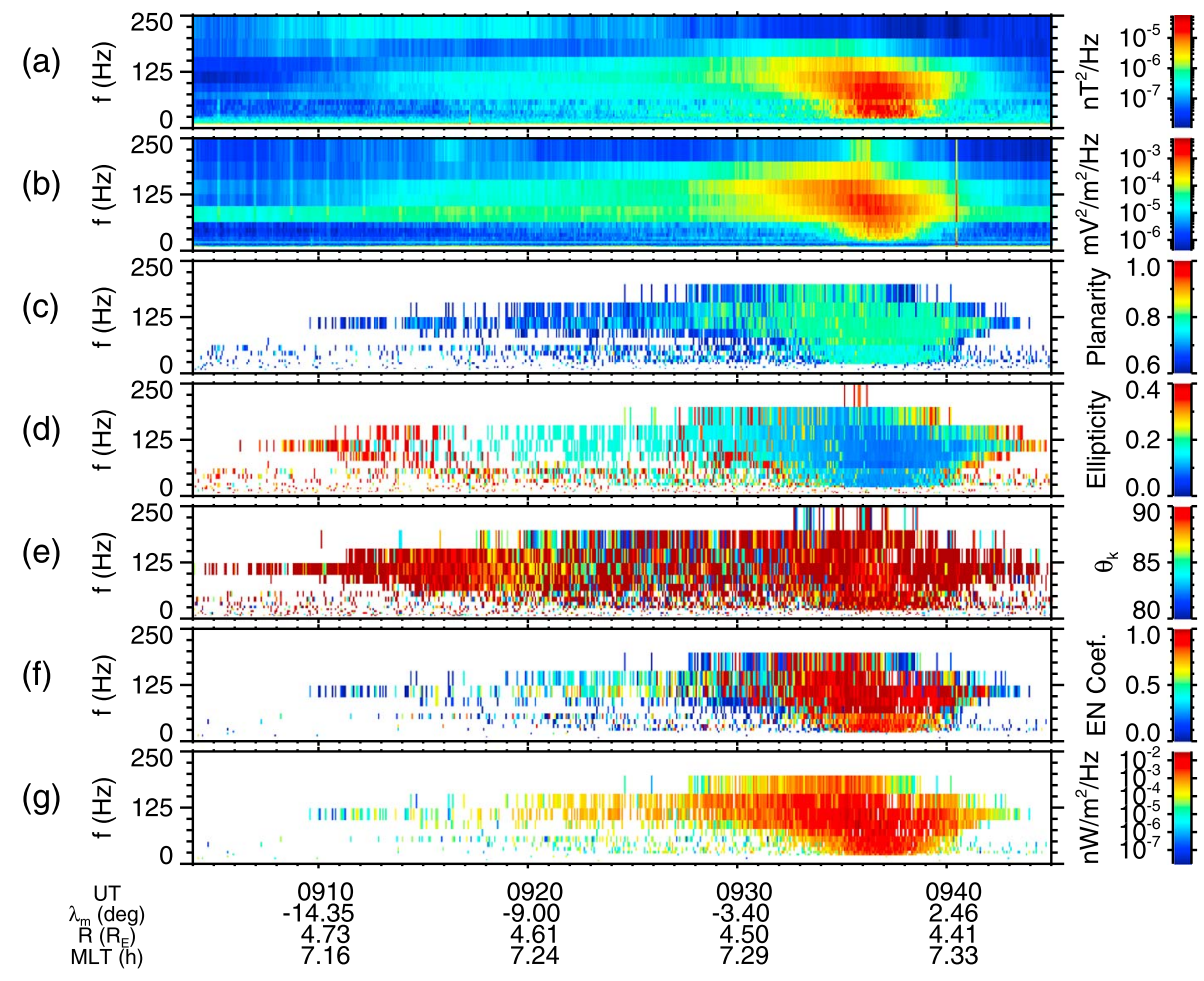

Figure 2. Same as Figure 1 but for the observations performed by Cluster 2 between 0904:00 and 0945:00 UT.

logic, which allows us to say that EN is observed in a given frequency-time interval with a certain probability (EN coefficient), i.e., a real number between 0 and 1. The EN coefficient equal to 0 would correspond to the situation of EN being completely absent, and the EN coefficient equal to 1 would correspond to the situation of EN being $100 \%$ present.

Taking into account the aforementioned thresholds for EN polarization and propagation parameters, we can define three partial EN coefficients. The first coefficient is related to the planarity of magnetic field fluctuations. If the planarity is larger than 0.8 , the planarity coefficient is equal to 1 . If the planarity is lower than 0.6 , the planarity coefficient is equal to 0 . If the planarity is between 0.6 and 0.8 , a linear transition function is used. The same approach is used for the remaining two coefficients. Namely, the second coefficient is related to the ellipticity of magnetic field fluctuations. If the ellipticity is lower than 0.2 , the ellipticity coefficient is equal to 1 . If the ellipticity is larger than 0.4 , the ellipticity coefficient is equal to 0 . If the ellipticity is between 0.2 and 0.4 , a linear transition function is used. The third coefficient is related to the wave normal angle. If the wave normal angle is larger than $85^{\circ}$, the wave normal coefficient is equal to 1. If the wave normal angle is lower than $80^{\circ}$, the wave normal coefficient is equal to 0 . If the wave normal angle is between 80 and $85^{\circ}$, a linear transition function is used. The resulting EN coefficient is calculated as the product of the three partial coefficients. This final EN coefficient is presented in Figures $1 f$ and $2 f$. Only frequency-time intervals with nonzero values of EN coefficients are plotted. It can be seen that the resulting frequency-time intervals with nonzero EN coefficients correspond well to the frequency-time intervals of EN noise emissions, which can be intuitively identified according to the data plotted in the preceding panels. We note that possible frequency-time intervals which contain electrostatic emissions but no EN are also excluded by this data processing, as they are not accompanied by a magnetic signal, and they thus do not have the required polarization properties of magnetic field fluctuations.

Figures $1 \mathrm{~g}$ and $2 \mathrm{~g}$ show the frequency-time plots of estimated EN Poynting flux spectral density. Although the multicomponent measurements performed by the Cluster spacecraft allow us in most cases to evaluate the Poynting flux as the time-averaged vector product of the electric and magnetic field, its calculation is in this case subject to large experimental uncertainties due to the peculiarities of EN polarization properties [Němec et al., 2013]. The Poynting flux spectral density was therefore calculated from the measured power spectral densities of magnetic and electric field fluctuations and from the magnitude of the ambient 
magnetic field using the theory of electromagnetic waves in the cold plasma approximation [Stix, 1992]. Wave vector directions exactly perpendicular to the ambient magnetic field are assumed in the calculation. According to the theory of electromagnetic waves in the cold plasma approximation, this corresponds to the magnetic field fluctuations linearly polarized along the ambient magnetic field, and the electric field fluctuations elliptically polarized in the plane perpendicular to the ambient magnetic field, with the main polarization axis oriented along the wave vector direction. This knowledge of theoretical polarization properties allows us to properly evaluate the Poynting flux using the two available electric field components, with no need for the third electric field component to be measured. We determine the plasma number density at the observation point and the ellipticity of electric field fluctuations from the condition that the theoretically calculated $B$-to- $E$ ratio corresponds to the measured $B$-to- $E$ ratio. This was successfully used by Němec et al. [2006], who demonstrated that the calculated plasma number densities are in agreement with plasma number densities determined from the spacecraft potential, with most probable relative error being around $30 \%$. Having determined the polarization properties of the observed emissions, the Poynting flux spectral density can be evaluated directly from the square root of the product of power spectral densities of electric and magnetic field fluctuations, accounting for a factor due to the wave polarization. As we are interested in the Poynting flux spectral density of exclusively EN emissions, the calculated Poynting flux spectral density estimates are multiplied by the EN coefficients from Figures $1 \mathrm{f}$ and $2 \mathrm{f}$.

We note that if EN emissions occurred in the same frequency-time intervals with electrostatic emissions, these intervals would be included in the analysis, and the procedure would result in an underestimation of the plasma number density. However, taking into account that the obtained plasma number densities agree reasonably well with the observations [Nèmec et al., 2006], this is likely an exceptional case and it is not important for the performed statistical study. We also note that due to successive failures of different Electric Field and Wave (EFW) probes, the power spectral density of electric field fluctuations can be underestimated by a factor of up to 2 [Cornilleau-Wehrlin et al., 2014]. This necessarily influences both the obtained values of Poynting flux and the calculated plasma number densities. However, the impacts of the failures of EFW probes are greatly reduced by the large statistical study, and they thus do not affect the obtained overall results.

Having evaluated the EN Poynting flux spectral density in all relevant frequency-time intervals, we can determine the intensity of a given EN event at any given frequency as the EN Poynting flux spectral density integrated over the geomagnetic latitude. For each EN event, this integration is performed within the latitudinal range corresponding to the beginning and ending time of the event according to the list of Hrbáčková et al. [2015]. We can further determine the total intensity of a given EN event as the EN Poynting flux spectral density integrated over the geomagnetic latitude and the frequency (up to $500 \mathrm{~Hz}$, which is the upper estimate of the lower hybrid frequency throughout the data set). The resulting total intensity of the EN event from Figure 1 is $11.3 \mathrm{nW} \mathrm{deg} / \mathrm{m}^{2}$. The resulting total intensity of the EN event from Figure 2 is $3.8 \mathrm{nW} \mathrm{deg} / \mathrm{m}^{2}$.

\section{Results}

Figure 3a shows the median Poynting flux spectral density of EN events as a function of frequency. The results obtained for EN events observed in the plasmasphere are shown in blue, whereas the results obtained for EN observed in the plasma trough are shown in red. These regions were classified according to the approach used by Němec et al. [2013], i.e., the regions with the estimated plasma number density larger than $100 \mathrm{~cm}^{-3}$ were classified as the plasmasphere (1047 EN events), and the regions with the estimated plasma number density lower than $30 \mathrm{~cm}^{-3}$ were classified as the plasma trough (638 EN events). Note that the regions with plasma number densities between 30 and $100 \mathrm{~cm}^{-3}$ are classified neither as the plasmasphere nor as the plasma trough, and the corresponding EN events are not included in the analysis when this region classification is used. It can be seen that the median Poynting flux spectral density of EN events observed in the plasma trough is approximately the same as the median Poynting flux spectral density of EN events observed in the plasmasphere. Moreover, EN events observed in the plasma trough generally occur at higher frequencies and have larger frequency bandwidths. This might be partly explained by the sampling bias. Namely, the Cluster spacecraft are located in the plasma trough preferentially during the times of the compressed plasmasphere. Assuming that the generation region is located close to the plasmapause [Nèmec et al., 2013], this would imply higher cyclotron frequencies in the source region, i.e., the same harmonic numbers would correspond to higher frequencies. 
(a)

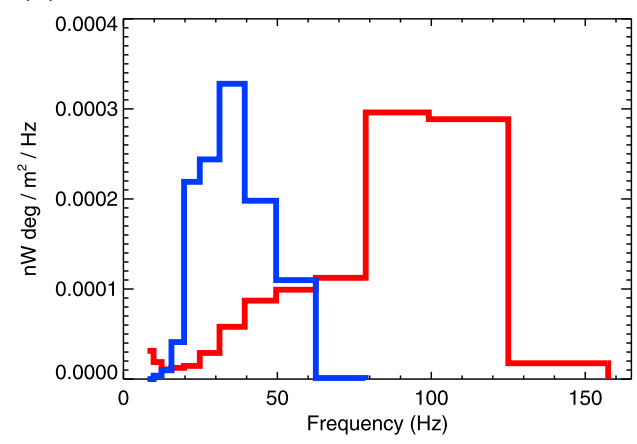

(b)

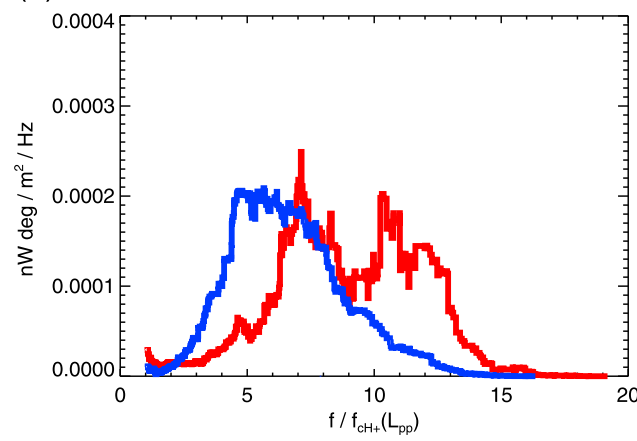

Figure 3. (a) Median Poynting flux spectral density of EN events as a function of frequency. (b) Median Poynting flux spectral density of EN events as a function of frequency normalized by the proton cyclotron frequency at the model plasmapause location. The results obtained for EN observed in the plasmasphere are shown in blue, whereas the results obtained for EN observed in the plasma trough are shown in red.

We therefore attempt to normalize the frequencies by the proton cyclotron frequency at the plasmapause location. The results obtained using the model plasmapause location [Moldwin et al., 2002] are shown in Figure 3b. It can be seen that the normalized frequencies of EN events observed in the plasmasphere and in the plasma trough are closer to each other than the frequencies before the normalization. However, the normalized frequencies of EN events observed in the plasma trough are still significantly higher than the normalized frequencies of EN events observed in the plasmasphere. We note that only the frequency bins with at least $25 \%$ data coverage are plotted in Figure $3 \mathrm{~b}$. We also note that qualitatively the same results are obtained when EN frequencies are normalized by the proton cyclotron frequencies at observation points rather than by the proton cyclotron frequencies at the model plasmapause locations. However, the frequencies of EN events observed in the plasmasphere and in the plasma trough normalized by the proton cyclotron frequencies at observation points differ more substantially than with the used normalization, resembling more the non-normalized distribution in Figure 3a (not shown). This indicates that the used normalization by the proton cyclotron frequencies at the plasmapause locations is more valid than the normalization by the proton cyclotron frequencies at observation points, consistently with the preferred source region located close to the plasmapause [Němec et al., 2013], and a significant radial distance that the waves can propagate from the generation region to the observation point [Horne et al., 2000; Santolik et al., 2002].

Figures $4 \mathrm{a}$ and $4 \mathrm{~b}$ show the total intensities of individual $\mathrm{EN}$ events as a function of magnetic local time (MLT) in the plasmasphere and in the plasma trough regions, respectively. The overplotted thick solid lines correspond to median values, and the thinner solid lines correspond to 0.25 and 0.75 quartiles. The MLT dependencies inside the plasmasphere and in the plasma trough are slightly different. The median total intensity of EN events observed in the plasmasphere is nearly independent on MLT. The intensities might be

(a)

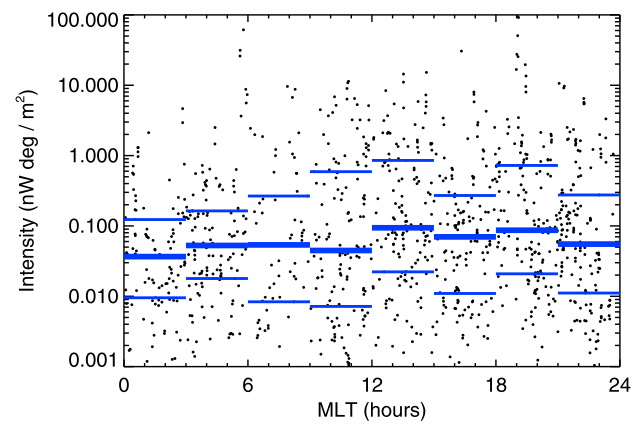

(b)

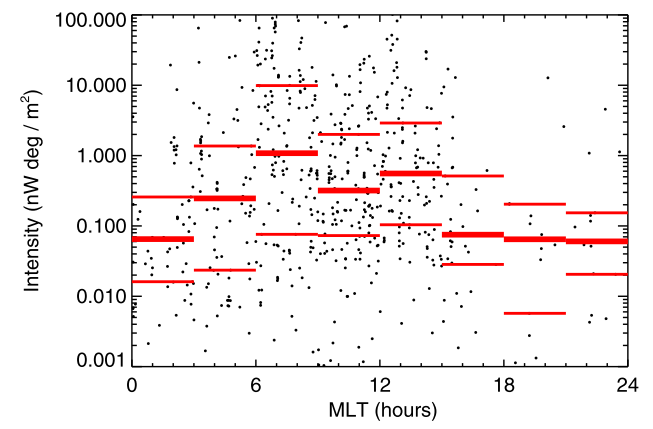

Figure 4. Total intensity of individual EN events as a function of magnetic local time. Thick solid lines correspond to the median dependencies, and thinner solid lines correspond to 0.25 and 0.75 quartiles. (a) EN events observed in the plasmasphere. (b) EN events observed in the plasma trough. 
(a)

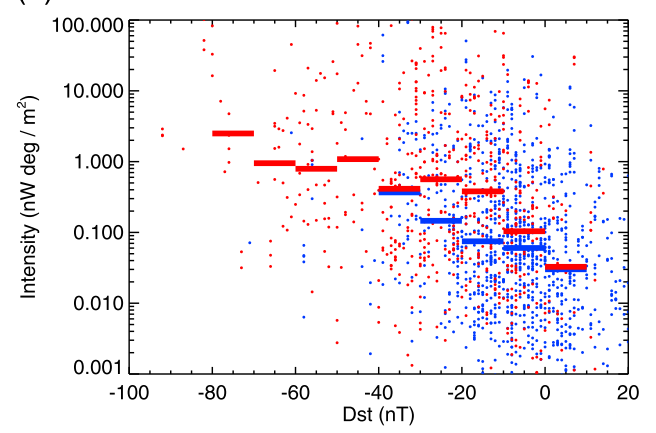

(b)

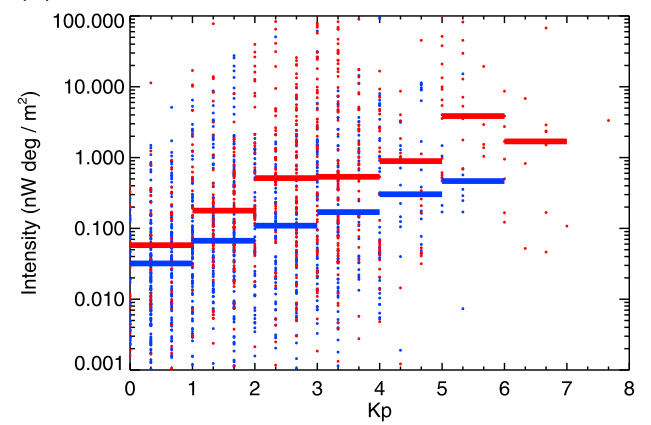

Figure 5. (a) Total intensity of individual EN events as a function of Dst index at the time of the measurement. (b) Total intensity of individual EN events as a function of $\mathrm{Kp}$ index at the time of the measurement. Solid lines correspond to median values over a given interval. The results obtained for EN events observed in the plasmasphere are shown in blue, whereas the results obtained for EN events observed in the plasma trough are shown in red.

possibly somewhat larger in the dusk sector than in the dawn sector, but taking into account the scatter of the data points, this trend is not very significant. On the other hand, the median total intensity of EN events observed in the plasma trough has a broad maximum in the noon sector.

The total intensity of EN events as a function of $D s t$ and $K p$ geomagnetic indices is analyzed in Figures $5 \mathrm{a}$ and $5 \mathrm{~b}$, respectively. The results obtained for $\mathrm{EN}$ events inside the plasmasphere and in the plasma trough are plotted by blue and red colors, respectively. In order to better visualize the overall trend, the median values calculated over given intervals of $D s t$ and $K p$ with at least 10 data points are overplotted by the solid lines. Although the scatter of the data point is rather large, it can be seen that the total intensity of EN events observed in the plasma trough is on average slightly larger than the total intensity of EN events observed in the plasmasphere. Moreover, the intensity of EN events both inside the plasmasphere and in the plasma trough increases with the geomagnetic activity.

Since Cluster is a multispacecraft mission, we can use the observations of the same EN event by several different Cluster spacecraft to evaluate the spatiotemporal variability of EN emissions [Němec et al., 2005]. The temporal variability provides important information about time scales on which the conditions in the generation region vary. On the other hand, the interpretation of the spatial variability of EN emissions is more difficult, as it combines the information about the source size with the wave propagation, which likely plays a dominant role. The approach that we have used is based on comparing the data measured by each pair of Cluster spacecraft. Note that with four Cluster spacecraft we have six pairs for a given time. The intensity of EN emissions is controlled primarily by the geomagnetic latitude [Němec et al., 2005]. We will therefore compare latitudinal dependencies rather than time dependencies. Following the approach used for determining total intensities of EN events, we do not impose any explicit latitudinal limits on the analyzed events. Instead, for each EN event, we use the latitudinal range corresponding to the beginning and ending time of the event according to the list of Hrbácková et al. [2015].

Figure 6a shows the latitudinal dependencies corresponding to the EN event from Figures 1 and 2 . The red curve shows the data for Cluster 1; i.e., it corresponds to Figure 1. The blue curve shows the data for Cluster 2; i.e., it corresponds to Figure 2. Latitudinal dependence of EN Poynting flux spectral densities is evaluated in frequency channels up to $500 \mathrm{~Hz}$ (the upper estimate of the lower hybrid frequency throughout the data set), i.e., in the 18 lowest frequency channels of the instrument. However, for the purpose of representing the data in a single plot in Figure 6a we have used the Poynting flux spectral densities integrated over the 18 frequency channels. The resulting latitudinal dependence is plotted in the first panel. The second panel shows the latitudinal dependence of the time in minutes after 0900:00 UT. The third panel of Figure 6a shows the latitudinal dependence of the radial distance of the Cluster spacecraft, and the fourth panel shows the latitudinal dependence of the spacecraft MLT.

The latitudinal dependencies of the sum of Poynting flux spectral densities measured by Cluster 1 and Cluster 2 shown in Figure 6a are rather similar. However, Cluster 1 observes slightly larger intensities, reached at somewhat larger geomagnetic latitudes. This latitudinal shift might be possibly due to the nondipole geometry of the real magnetic field configuration. Namely, Němec et al. [2006] used more realistic 
(a)

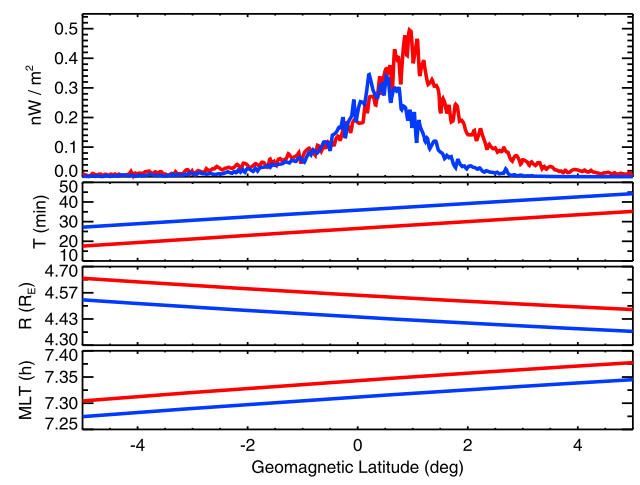

(b)

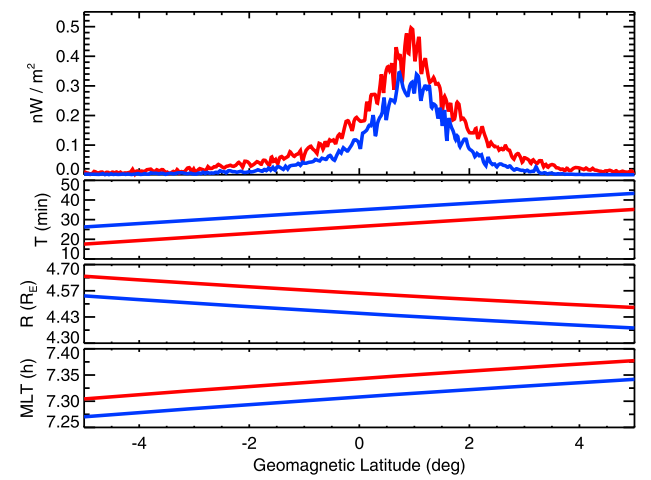

Figure 6. Latitudinal variation of selected EN parameters corresponding to the example EN event from Figures 1 and 2. Red curve shows the data for Cluster 1; i.e., it corresponds to Figure 1. Blue curve shows the data for Cluster 2; i.e., it corresponds to Figure 2. Latitudinal dependence of the sum of Poynting flux spectral densities over individual frequency channels of the STAFF-SA instrument is shown in the first panels. Latitudinal dependence of the time in minutes after 0900:00 UT is shown in the second panels. The third panels show the latitudinal dependence of the radial distance of the Cluster spacecraft. The fourth panels show the latitudinal dependence of the MLT of the Cluster spacecraft. (a) Latitudinal dependencies of actually measured data. (b) Latitudinal dependencies shifted to the position of the maximum correlation.

magnetic field models to determine the true $(\min -B)$ magnetic equator, and they reported that most intensity peaks of EN occur exactly at the min- $B$ magnetic equator. We therefore shift the latitudinal dependencies from Figure 6a in order to obtain the maximum correlation between the Poynting flux spectral densities. The correlations are evaluated using the data from all 18 frequency channels separately. Only the frequency-latitude intervals where at least one of the spacecraft observed a nonzero EN Poynting flux spectral density were used for the calculation of the correlation.

The optimal latitudinal shift corresponding to Figure $6 a$ was found to be about $0.52^{\circ}$, and the resulting value of the correlation coefficient is equal to about 0.85 . The shifted latitudinal dependencies are shown in Figure $6 \mathrm{~b}$. The red curves are the same as in Figure $6 \mathrm{a}$, while the blue curves were shifted to higher latitudes by $0.52^{\circ}$. The time delay and the spatial separation between the EN observations by Cluster 1 and Cluster 2 can be determined directly from the second, third, and fourth panels. The orbits of the Cluster spacecraft are very similar; i.e., the latitudinal dependencies of time, radial distance, and MLT for a pair of Cluster spacecraft are generally nearly parallel. The time delay between EN observations is then determined as the mean time difference of the curves in the second panel of Figure $6 \mathrm{~b}$ calculated over the event duration. Similarly, the radial distance separation of EN observations is determined as the mean difference of the curves in third panel of Figure $6 \mathrm{~b}$, and the MLT separation is determined as the mean difference of the curves in the fourth panel of Figure $6 \mathrm{~b}$. In the analyzed example event observed by Cluster 1 and Cluster 2 the time difference is about $8.7 \mathrm{~min}$, the radial distance separation is about $0.1 R_{E}$, and the MLT separation is about $0.03 \mathrm{~h}$.

We have used this procedure for all EN events observed by at least two Cluster spacecraft. If an event was observed by more than two Cluster spacecraft, all possible spacecraft pairs were considered separately. Altogether, 2122 relevant pair events were identified in the analyzed data set. For each of them we have evaluated the value of the correlation coefficient, ratio of the event intensities, time delay, radial distance separation, and MLT separation. The aim is to determine how the correlation coefficients vary as a function of the time delay and the spatial separation between the spacecraft. Unfortunately, as the sampled time delays and the spatial separations are given by the spacecraft orbits, only data obtained in a limited interval of radial distances and MLT separations are typically available. This is the principal limitation of the analyzed data set which cannot be overcome.

The spatial variability of EN emissions is analyzed in Figure 7. In order to minimize the influence of the temporal variability of the emissions, we have limited the analysis only to the observations with a time delay lower than $0.1 \mathrm{~h}$. Figure 7a shows the correlation coefficient between a pair of EN observations as a function of their MLT separation. Figure 7b shows the correlation coefficient between a pair of EN observations as a function of their radial distance separation. An additional condition was imposed in each plot to ensure that the analyzed parameter should be the main controlling quantity in a given plot. Namely, only the events 
(a)

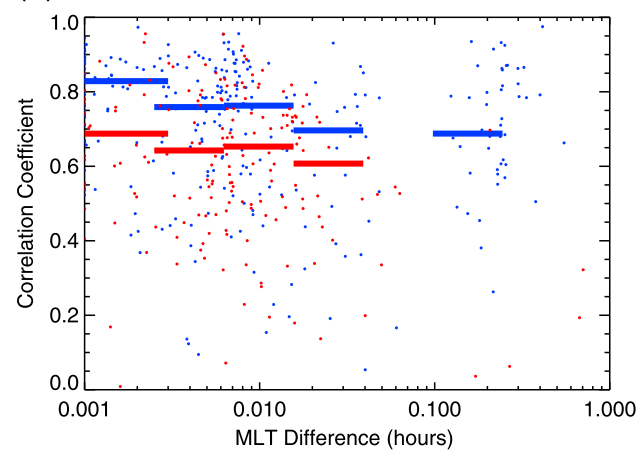

(b)

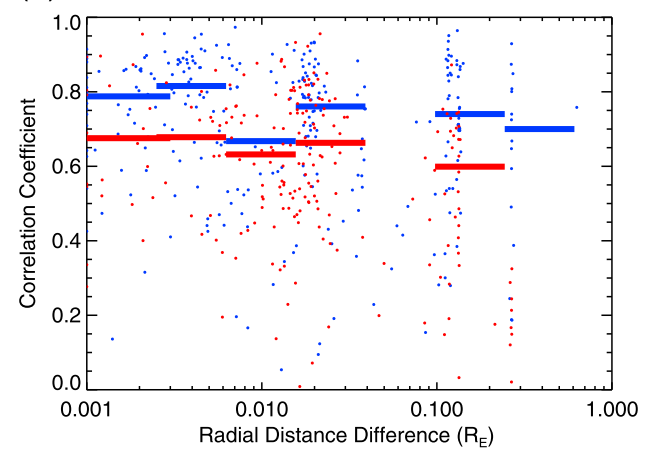

Figure 7. (a) Correlation coefficient between a pair of EN observations as a function of their magnetic local time separation. (b) Correlation coefficient between a pair of EN events as a function of their radial distance separation. The results obtained for EN event pairs observed in the plasmasphere are shown in blue, whereas the results obtained for EN event pairs observed in the plasma trough are shown in red. Solid horizontal lines correspond to median values over a given interval.

with the radial distance separation less than $0.1 R_{E}$ were considered in Figure $7 \mathrm{a}$, and only the events with the MLT separation less than $0.1 \mathrm{~h}$ were considered in Figure $7 \mathrm{~b}$. The results obtained for EN pairs observed in the plasmasphere are shown in blue, whereas the results obtained for EN pairs observed in the plasma trough are shown in red. The solid horizontal lines correspond to median values calculated over given intervals. It can be seen that the correlation coefficients do not significantly change over the analyzed MLT and radial distance separations. This is true also for the ratios of total intensities of EN events, which do not seem to depend on the spatial separation of the spacecraft (not shown). However, we note once again that the sampled range of spatial separations is extremely limited due to the similar spacecraft orbits. Namely, principally no data are available for MLT separations larger than about $0.2 \mathrm{~h}$ and radial distance separations larger than about $0.2 R_{E}$. It is also noticeable that the median correlation coefficients obtained for event pairs in the plasma trough are typically lower than the correlation coefficients obtained for event pairs in the plasmasphere.

The temporal variability of EN emissions is analyzed in Figure 8a. The format of the figure is the same as the format used in Figure 7. In order to minimize the influence of the spatial variability of the emissions, we have limited the analysis to the event pairs closely separated in space $\left(\Delta \mathrm{MLT}<0.1 \mathrm{~h}, \Delta r<0.1 R_{E}\right)$. The values of the correlation coefficients obtained for event pairs in the plasmasphere are rather high for time delays lower than about $0.1 \mathrm{~h}$. At larger time delays their median values systematically decrease, and there is principally no correlation for time delays on the order of hours. The values of correlation coefficients

(a)

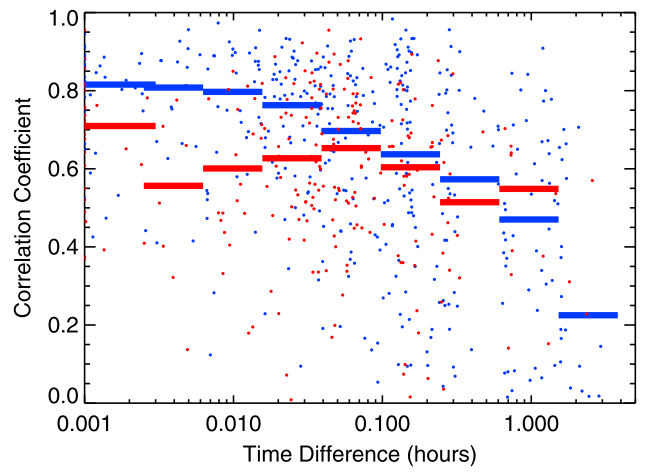

(b)

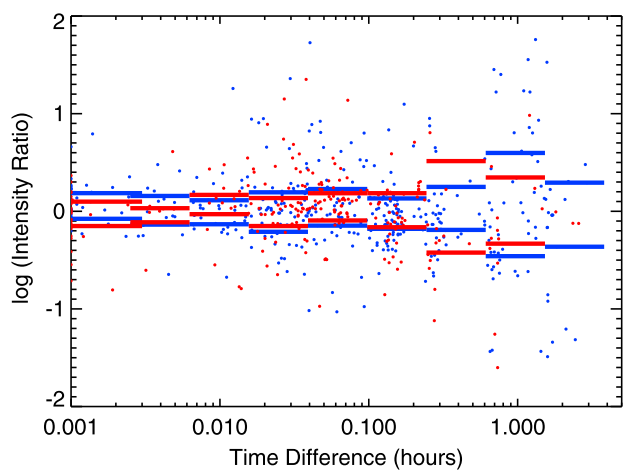

Figure 8. (a) Correlation coefficient between a pair of EN observations as a function of their time difference. The used format is the same as in Figure 7. (b) Logarithm of ratios of intensities of EN events as a function of their time difference. The ratios of intensities larger than 1 correspond to EN intensities at later times being larger, whereas the ratios of intensities lower than 1 correspond to EN intensities at later times being lower. Horizontal lines correspond to median values calculated over given intervals from values of intensity ratios lower/larger than 1 separately. 
obtained for event pairs in the plasma trough are generally lower, and the trend of decreasing correlation with increasing time delay is less clear. The temporal variability of the ratio of total EN intensities is shown in Figure 8b. The results obtained for EN pairs observed in the plasmasphere are shown in blue, whereas the results obtained for EN pairs observed in the plasma trough are shown in red. The ratios of intensities larger than 1 correspond to EN intensities at later times being larger, whereas the ratios of intensities lower than 1 correspond to EN intensities at later times being lower. The horizontal lines correspond to median values calculated over given intervals from values of intensity ratios lower/larger than 1 separately. While most of the intensity ratios are close to 1 at low time delays, the scatter becomes rather high at time delays on the order of an hour.

\section{Discussion}

The exact frequency-time intervals of EN emissions were determined using the analysis of wave propagation parameters, rather than using only the electric/magnetic field intensity and the frequency range. This approach was shown to be very efficient in distinguishing EN events from other natural emissions [Santolik et al., 2004; Němec et al., 2005, 2006, 2013; Hrbáčková et al., 2015]. Moreover, its extension using the fuzzy classification of the EN presence is readily applicable for determining the overall intensity of EN emissions. There is, however, an additional important point which was often not properly considered in previous EN studies (and studies of wave intensity, in general). Namely, the wave intensity in the proper sense is equal to the Poynting flux of the emissions, not exclusively to the magnetic field intensity and neither exclusively to the electric field intensity. As $B$-to- $E$ ratio depends on the refractive index, evaluating the intensity of the emissions using only the magnetic or only on the electric field data can lead to systematic errors. Unfortunately, due to the peculiar polarization properties of EN emissions, it is not possible to evaluate their Poynting flux directly using the available data [Němec et al., 2013].

However, the used calculation based on the theory of electromagnetic waves in cold plasma approximation allowed us to evaluate the Poynting flux properly from the measured magnetic and electric spectral densities and the magnitude of the ambient magnetic field. The usage of the Poynting flux as a characterization of the wave intensity instead of exclusively the electric or exclusively the magnetic field intensity represents major advantage as compared to most former studies. The main reason is that although it may slightly complicate the usage in diffusion codes (which require wave magnetic field intensities), it allows us to efficiently compare wave intensities in different density regions, which would be otherwise impossible. The calculation further allowed us to determine local plasma number densities from EN observations [Němec et al., 2006]. Following the density threshold criteria used by Němec et al. [2013], the calculated plasma number densities were used to determine whether a given EN event was observed inside the plasmasphere or in the plasma trough. This is important, because the plasmapause boundary plays a crucial role for EN emissions, as was demonstrated by the analysis of their propagation directions [Chen and Thorne, 2012; Němec et al., 2013] and the analysis of their occurrence rate [Hrbáčková et al., 2015].

When analyzing EN properties in the plasmasphere and in the plasma trough, one should keep in mind the sampling bias that stems directly from the used data coverage. Namely, the Cluster spacecraft sample the equatorial plane usually at approximately the same radial distance of $4 R_{E}$, i.e., close to the mean plasmapause location. EN events observed by the Cluster spacecraft in the plasma trough occurred therefore preferentially at times when the plasmapause was at lower radial distances. Since the plasmapause moves inward during geomagnetically disturbed periods, this corresponds to the periods of enhanced geomagnetic activity. We note that although this was the case for most of the analyzed period, the change of the Cluster orbits since 2008 resulted in sampling the equatorial plane at both lower and larger radial distances. This was, coincidentally, also the period of solar minimum. Then it is not possible in our data set to unambiguously separate the effect of different radial distances and different solar activity. Nevertheless, the EN occurrence rates reported at these lower and larger radial distances were generally much lower than the occurrence rates reported at radial distances of about $4 R_{E}$ [Hrbáčková et al., 2015]. However, one should keep in mind that there is a relation between the sampled radial distances and the level of the geomagnetic activity, which likely acts to strengthen the dependencies obtained in Figure 5, namely, that the intensity increases with the geomagnetic activity.

Our analysis shows that the total intensities of EN events observed in the plasma trough are on average slightly higher than the total intensities of EN events observed in the plasmasphere. This could be related 
to the aforementioned sampling bias. Since the plasma trough events are observed preferentially during periods of enhanced geomagnetic activity, which favors larger intensities of EN events [Meredith et al., 2008; Ma et al., 2013], larger intensities of EN events observed in the plasma trough than in the plasmasphere might be expected. However, the results from Figure 5 show that the median EN intensity in the plasma trough is higher than the median EN intensity in the plasmasphere for all analyzed levels of the geomagnetic activity. Nevertheless, it should be noted that the MLT dependence of the plasmapause location leads to another sampling bias of the used data set. As pointed out by Moldwin et al. [2002], the average plasmapause on the dayside is by up to $1 R_{E}$ closer to the Earth than on the nightside. Consequently, EN events in the plasma trough are observed preferentially in the noon sector, where their intensity is the largest.

The largest intensity of plasma trough EN events in the noon sector is in agrement with the results of the EN occurrence rate analysis performed by Hrbáčková et al. [2015]. These results strongly suggest that EN in the plasma trough is generated preferentially at these MLTs. However, the MLT dependence of the intensity of EN events observed in the plasmasphere is rather different. There seems to be only a weak dependence on MLT. This is likely in agreement with the occurrence rate results presented by Hrbáčková et al. [2015] in their Figure $8 c$, where the occurrence rate can be identified as almost independent on MLT. Following the discussion of Hrbáčková et al. [2015] and the raytracing results of Chen and Thorne [2012], we believe that the reason that the MLT dependence of EN intensity inside the plasmasphere is only very weak is the effective trapping of the emissions inside the plasmasphere and their ability to travel over a broad range of MLTs.

EN events observed in the plasma trough have higher frequencies than EN events observed in the plasmasphere. This might be partly explained by the aforementioned sampling bias, assuming that the generation region is located close to the plasmapause [Němec et al., 2013]. Nevertheless, even after the normalization of the observed EN frequencies by the proton cyclotron frequencies at the model plasmapause locations, the normalized frequencies of EN events observed in the plasma trough are higher than the normalized frequencies of EN events observed in the plasmasphere. Specifically, while the harmonic numbers of EN events observed inside the plasmasphere typically range from about 3 to 10 , the harmonic numbers of EN events observed in the plasma trough typically range from about 5 to 13 . This indicates that the frequency difference is a real effect. We believe that it might be consistent with the simulation results by Chen et al. [2010] and Ma et al. [2014], who reported that lower frequency waves are generated in higher-density regions, while higher-frequency waves are generated in lower density regions. However, the theoretically predicted difference in the harmonic numbers of EN events excited inside the plasmasphere and in the plasma trough [Chen et al., 2010] is larger than the one we observe. Nevertheless, one should consider that EN can travel a significant radial distance between the source region and the observation point [Horne et al., 2000; Santolík et al., 2002]; i.e., the classification based on the region where the waves were observed likely mixes waves generated in different regions together, smoothing out the differences between the two distributions. It is also noteworthy that the size of the spatial region inside the plasmasphere where the waves can be trapped decreases with increasing wave frequency [Chen and Thorne, 2012], which is an additional mechanism for preferential observation of lower frequency EN events in the plasmasphere. Finally, we note that according to Ma et al. [2014], no wave excitation inside the plasmasphere is possible, and the waves observed in there are likely to propagate from a source just outside the plasmasphere.

Multipoint measurements performed by the Cluster spacecraft allow us to analyze the spatiotemporal variability of EN emissions. Unfortunately, the spatial separations of the Cluster spacecraft in the equatorial region ( $\Delta \mathrm{MLT}<0.2 \mathrm{~h}, \Delta r<0.2 R_{E}$ ) are generally lower than typical spatial scales of the EN variability, which does not allow their proper analysis. This is in agreement with the results obtained by Nermec et al. [2005], who evaluated the ratios of the peak amplitudes of EN emissions as a function of their separation in the equatorial plane. Nermec et al. [2005] also reported that the variations of the ratios of EN peak amplitudes increase with a time delay between the measurements in an interval from tenths to hundreds of minutes. Our more detailed analysis of EN temporal variability based on the correlation analysis is in agreement with these former results, at least for EN events observed in the plasmasphere. For EN events observed in the plasma trough the situation is less clear. The values of correlation coefficients are generally lower than in the plasmasphere, which is likely in agreement with the absence of EN trapping in the plasma trough region [Chen and Thorne, 2012]. The absence of EN trapping might also possibly explain the unclear trend of the correlation coefficient dependence. Assuming that EN is generated simultaneously at several locations, it 
would be seen as one homogenous EN event in the plasmasphere, while the situation in the plasma trough would be necessarily significantly more variable. Nevertheless, on time scales of about an hour the situation inside the plasmasphere and in the plasma trough becomes comparable, indicating that this is the typical time scale corresponding to the change of the source properties.

\section{Conclusions}

More than $2000 \mathrm{EN}$ events identified in the Cluster spacecraft data during the years 2001-2010 were analyzed. Frequency-time intervals containing EN emissions were identified using fuzzy logic based on wave propagation parameters, which allows us to distinguish EN events from other emissions. The analysis of $B$-to- $E$ ratios of the observed emissions allowed us to evaluate the plasma number density at the spacecraft location, which was used to classify the measurements according to whether they were obtained inside or outside the plasmasphere. The agreement between the obtained plasma number densities and the plasma number densities determined from the spacecraft potential [Němec et al., 2006] further shows that the number of frequency-time intervals containing both a significant level of electrostatic emissions and EN (and thus passing our selection criteria) is small and not important for the performed statistical study. The total intensity of individual EN events was determined based on the evaluation of the Poynting flux rather than on the evaluation of only the electric/magnetic field intensity.

It is found that EN emissions have higher frequencies and on average larger total intensities in the plasma trough than in the plasmasphere. EN events observed in the plasma trough are most intense close to the local noon, while EN events observed in the plasmasphere are nearly independent on MLT. The intensity of EN events is enhanced during disturbed periods, both inside the plasmasphere and in the plasma trough. Our results are consistent with the wave generation in the plasma trough, but a possibility of a part of the waves being generated in the plasmasphere cannot be excluded. Observations of the same EN events by several Cluster spacecraft allowed us to estimate their spatiotemporal variability. EN emissions observed in the plasmasphere do not change on the analyzed spatial scales $\left(\Delta \mathrm{MLT}<0.2 \mathrm{~h}, \Delta r<0.2 R_{E}\right.$ ), but they change significantly on time scales of about an hour. The same appears to be the case also for EN events observed in the plasma trough, although the plasma trough dependencies are less clear.

\section{Acknowledgments}

The data used in this study can be obtained from the Cluster Science Archive (CSA) (http://www.cosmos. esa.int/web/csa). We would like to thank the CSA and all the involved personnel. This work was supported by GACR grant 15-01775Y, KONTAKT II grants LH14010 and LH11122, and GAUK grant 678212.

Michael Liemohn thanks Nigel Meredith and another reviewer for their assistance in evaluating this paper.

\section{References}

Boardsen, S. A., D. L. Gallagher, D. A. Gurnett, W. K. Peterson, and J. L. Green (1992), Funnel-shaped, low-frequency equatorial waves, J. Geophys. Res., 97, 14,967-14,976.

Bortnik, J., and R. M. Thorne (2010), Transit time scattering of energetic electrons due to equatorially confined magnetosonic waves, J. Geophys. Res., 115, A07213, doi:10.1029/2010JA015283.

Chen, L., and R. M. Thorne (2012), Perpendicular propagation of magnetosonic waves, Geophys. Res. Lett., 39, L14102, doi:10.1029/2012GL052485.

Chen, L., R. M. Thorne, V. K. Jordanova, and R. B. Horne (2010), Global simulation of magnetosonic wave instability in the storm time magnetosphere, J. Geophys. Res., 115, A11222, doi:10.1029/2010JA015707.

Chen, L., R. M. Thorne, V. K. Jordanova, M. F. Thomsen, and R. B. Horne (2011), Magnetosonic wave instability analysis for proton ring distributions observed by the LANL magnetospheric plasma analyzer, J. Geophys. Res., 116, A03223, doi:10.1029/2010JA016068.

Cornilleau-Wehrlin, N., et al. (1997), The Cluster Spatio-Temporal Analysis of Field Fluctuations (STAFF) experiment, Space Sci. Rev., 79, $107-136$.

Cornilleau-Wehrlin, N., et al. (2003), First results obtained by the Cluster STAFF experiment, Ann. Geophys., 21, 437-456, doi:10.5194/angeo-21-437-2003.

Cornilleau-Wehrlin, N., R. Piberne, C. Lacombe, and S. Team (2014), User guide to the STAFF measurements in the Cluster Active Archive (CAA). Project: Cluster Active Archive, CAA-EST-UG-002(3.4).

Curtis, S. A., and C. S. Wu (1979), Gyroharmonic emissions induced by energetic ions in the equatorial plasmasphere, J. Geophys. Res., 84(A6), 2597-2607.

Gurnett, D. A. (1976), Plasma wave interactions with energetic ions near the magnetic equator, J. Geophys. Res., 81, $2765-2770$.

Horne, R. B., G. V. Wheeler, and H. S. C. K. Alleyne (2000), Proton and electron heating by radially propagating fast magnetosonic waves, J. Geophys. Res., 105(A12), 27,597-27,610.

Horne, R. B., R. M. Thorne, S. A. Glauert, N. P. Meredith, D. Pokhotelov, and O. Santolik (2007), Electron acceleration in the Van Allen radiation belts by fast magnetosonic waves, J. Geophys. Res., 34, L17107, doi:10.1029/2007GL030267.

Hrbáčková, Z., O. Santolík, F. Němec, E. Macúšová, and N. Cornilleau-Wehrlin (2015), Systematic analysis of occurrence of equatorial noise emissions using 10 years of data from the Cluster mission, J. Geophys. Res. Space Physics, 120, doi:10.1002/2014JA020268, in press.

Kasahara, Y., H. Kenmochi, and I. Kimura (1994), Propagation characteristics of the ELF emissions observed by the satellite Akebono in the equatorial plane, Radio Sci., 29, 751-767.

Laakso, H., H. Junginger, A. Roux, R. Schmidt, and C. de Villedary (1990), Magnetosonic waves above $f_{c H+}$ at geostationary orbit: GEOS 2 results, J. Geophys. Res., 95, 10,609-10,621.

$\mathrm{Li}$, J., et al. (2014), Interactions between magnetosonic waves and radiation belt electrons: Comparisons of quasi-linear calculations with test particle simulations, Geophys. Res. Lett., 41, 4828-4834, doi:10.1002/2014GL060461.

Liu, K., S. P. Gary, and D. Winske (2011), Excitation of magnetosonic waves in the terrestrial magnetosphere: Particle-in-cell simulations, J. Geophys. Res., 116, A07212, doi:10.1029/2010JA016372. 
Ma, Q., W. Li, R. M. Thorne, and V. Angelopoulos (2013), Global distribution of equatorial magnetosonic waves observed by THEMIS, Geophys. Res. Lett., 40, 1895-1901, doi:10.1002/grl.50434.

Ma, Q., W. Li, L. Chen, R. M. Thorne, and V. Angelopoulos (2014), Magnetosonic wave excitation by ion ring distributions in the Earth's inner magnetosphere, J. Geophys. Res. Space Physics, 119, 844-852, doi:10.1002/2013JA019591.

McClements, K. G., and R. O. Dendy (1993), Ion cyclotron harmonic wave generation by ring protons in space plasmas, J. Geophys. Res., $98,11,689-11,700$.

McClements, K. G., R. O. Dendy, and C. N. Lashmore-Davis (1994), A model for the generation of obliquely propagating ULF waves near the magnetic equator, J. Geophys. Res., 99, 23,685-23,693.

Meredith, N. P., R. B. Horne, and R. R. Anderson (2008), Survey of magnetosonic waves and proton ring distributions in the Earth's inner magnetosphere, J. Geophys. Res., 113, A06213, doi:10.1029/2007JA012975.

Moldwin, M. B., L. Downward, H. K. Rassoul, R. Amin, and R. R. Anderson (2002), A new model of the location of the plasmapause: CRRES results, J. Geophys. Res., 107(A11), 1339, doi:10.1029/2001JA009211.

Mourenas, D., A. V. Artemyev, O. V. Agapitov, and V. Karsnoselskikh (2013), Analytical estimates of electron quasi-linear diffusion by fast magnetosonic waves, J. Geophys. Res. Space Physics, 118, 3096-3112, doi:10.1002/jgra.50349.

Němec, F., O. Santolík, K. Gereová, E. Macúšová, Y. de Conchy, and N. Cornilleau-Wehrlin (2005), Initial results of a survey of equatorial noise emissions observed by the cluster spacecraft, Planet. Space Sci., 53, 291-298.

Němec, F., O. Santolík, K. Gereová, E. Macúšová, H. Laakso, Y. de Conchy, M. Maksimovic, and N. Cornilleau-Wehrlin (2006), Equatorial noise: Statistical study of its localization and the derived number density, Adv. Space Res., 37, 610-616.

Němec, F., O. Santolík, J. S. Pickett, Z. Hrbáčková, and N. Cornilleau-Wehrlin (2013), Azimuthal directions of equatorial noise propagation determined using 10 years of data from the Cluster spacecraft, J. Geophys. Res. Space Physics, 118, 7160-7169, doi:10.1002/2013JA019373.

Perraut, S., A. Roux, P. Robert, R. Gendrin, J. A. Sauvaud, J. M. Bosqued, G. Kremser, and A. Korth (1982), A systematic study of ULF waves above $f_{H_{+}}$from GEOS 1 and 2 measurements and their relationships with proton ring distributions, J. Geophys. Res., 87, 6219-6236.

Russell, C. T., R. E. Holzer, and E. J. Smith (1970), OGO 3 observations of ELF noise in the magnetosphere. The nature of the equatorial noise, J. Geophys. Res., 75(4), 755-768.

Santolík, O., J. S. Pickett, D. A. Gurnett, M. Maksimovic, and N. Cornilleau-Wehrlin (2002), Spatiotemporal variability and propagation of equatorial noise observed by Cluster, J. Geophys. Res., 107(A12), 1495, doi:10.1029/2001JA009159.

Santolík, O., M. Parrot, and F. Lefeuvre (2003), Singular value decomposition methods for wave propagation analysis, Radio Sci., 38(1), 1010, doi:10.1029/2000RS002523.

Santolík, O., F. Němec, K. Gereová, E. Macúšová, Y. de Conchy, and N. Cornilleau-Wehrlin (2004), Systematic analysis of equatorial noise below the lower hybrid frequency, Ann. Geophys., 22, 2587-2595.

Shprits, Y. Y., A. Runov, and B. Ni (2013), Gyro-resonant scattering of radiation belt electrons during solar minimum by fast magnetosonic waves, J. Geophys. Res. Space Physics, 118, 648-652, doi:10.1002/jgra.50108.

Stix, T. H. (1992), Waves in Plasmas, chap. Wave Normal Surfaces, Waves in a Cold Uniform Plasma, pp. 1-46, Springer, New York.

Tsurutani, B., B. J. Falkowski, J. S. Pickett, O. P. Verkhoglyadova, O. Santolík, and G. S. Lakhina (2014), Extremely intense ELF magnetosonic waves: A survey of polar observations, J. Geophys. Res. Space Physics, 119, 964-977, doi:10.1002/2013JA019284.

Xiao, F., Q. Zhou, Z. He, and L. Tang (2012), Three-dimensional ray tracing of fast magnetosonic waves, J. Geophys. Res., 117, A06208, doi:10.1029/2012JA017589.

Xiao, F., Q. Zhou, Z. He, C. Yang, Y. He, and L. Tang (2013), Magnetosonic wave instability by proton ring distributions: Simultaneous data and modeling, J. Geophys. Res. Space Physics, 118, 4053-4058, doi:10.1002/jgra.50401. 